\title{
An attempt to detect Vulcanoids with SOHO/LASCO images
}

\section{Scale relativity and quantization of the solar system}

\author{
G. Schumacher ${ }^{1}$ and J. Gay ${ }^{2}$ \\ 1 Observatoire de la Côte d'Azur, Département Augustin Fresnel, CNRS-UMR 6528, Av. Copernic, 06130 Grasse, \\ France \\ 2 Observatoire de la Côte d'Azur, Département Augustin Fresnel, CNRS-UMR 6528, Bd. de l'Observatoire, \\ BP 4229, 06304 Nice Cedex 4, France
}

Received 4 October 2000 / Accepted 28 November 2000

\begin{abstract}
We report an attempt to verify one of the predictions of Scale Relativity theory, namely that a small Vulcanoid should exist at 0.18 AU from the Sun. We have analyzed images taken by the coronograph LASCO aboard the satellite SOHO. Raw images are processed in order to eliminate cosmic ray impacts, stars and to improve the contrast. We have examined the resulting cleaned-up images both visually and by means of automatic detection using our own software. We have found no objects of visual magnitude 7 or brighter, corresponding to bodies with a diameter exceeding $60 \mathrm{~km}$.
\end{abstract}

Key words. gravitation - methods: data analysis - techniques: image processing - minor planets, asteroids solar system: general

\section{Introduction}

In a previous paper (Nottale et al. 1997) we gave a summary of the scale relativity theory, and we discussed its application to the solar system. Scale relativity assumes that the laws of physics are non-differentiable (Nottale 1993; Nottale 1996; Nottale 1997). Its main consequence is that gravitational forces tend to quantize the distribution of matter throughout the whole universe. Scale relativity theory can be seen as a generalization of quantum mechanics to the macroscopic world.

The theory predicts for the solar system that the positions of the planets are governed by:

$\sqrt{a} \simeq\left(n+\frac{1}{4}\right) \frac{\sqrt{G M}}{w_{0}}$

where $a$ is the semi-major axis of the orbit, $G$ the gravitational constant, $M$ the mass of the central object (the Sun), $w_{0}$ a fundamental constant $\left(=144 \mathrm{~km} \mathrm{~s}^{-1}\right)$ and $n$ an integer. Figure 1 shows the position of the planets versus the rank number $N=n-\frac{1}{4}$. One can see that in the inner solar system, the locations $N=1$ and $N=2$ are not occupied. They correspond to orbits with a semi-major axis of 0.05 and $0.18 \mathrm{AU}$, respectively (Fig. 1).

Send offprint requests to: G. Schumacher, e-mail: schumacher@obs-azur.fr

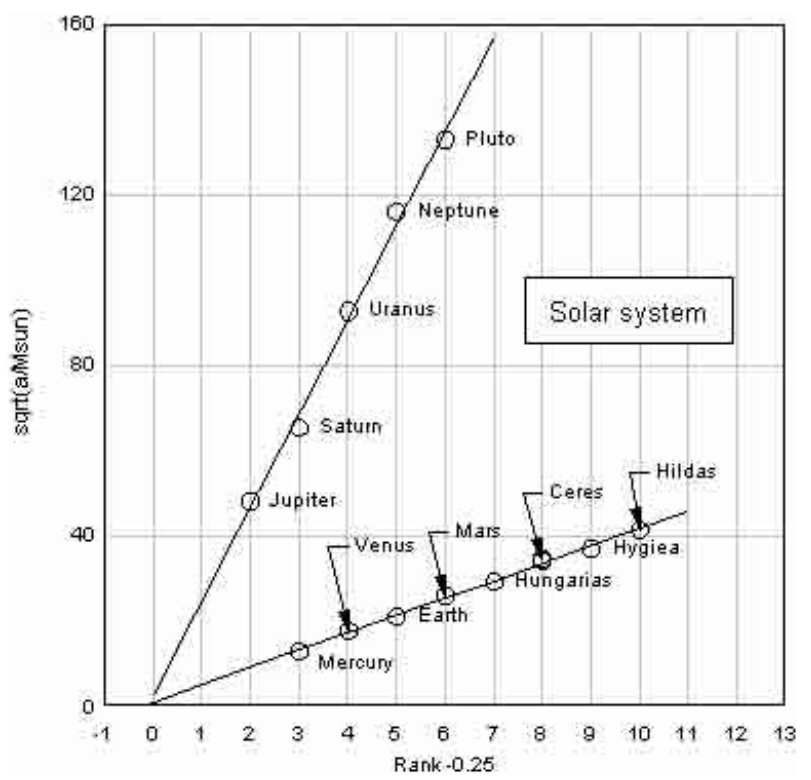

Fig. 1. Semi-major axis versus rank number $N$ for the planets of the inner and outer solar system. On the ordinate axis we have plotted the quantity $\sqrt{\frac{a}{M}}$ where $a$ is the semi-major axis in $\mathrm{km}$, and $M$ the mass of the Sun in Earth masses

Numerical models suggest that the area covered by those orbits $(N=1, N=2)$ is a stable region (Michel 1998; Evans 1999), and different authors (Lebofsky 1975; Vokrouhlicky 1999; Durda 2000) have shown that objects 
(so called "Vulcanoids") of the 10-km scale range and larger, can have survived in this region, since the formation of the solar system. We have also calculated that particles smaller than $0.2 \mu \mathrm{m}$ have been ejected from the solar environment by radiation pressure, and that objects smaller than $70 \mathrm{~m}$ have been attracted by the Sun due to the Poytning-Robertson effect. The scale relativity theory does not involve non-gravitational forces, but its basic statement is that for a chaotic system the distribution of matter is governed by Schrödinger-like equations over timescales longer than a Lyapunov time. The consequence is that, for a gravitational system for example, the matter has a tendency to concentrate on specific orbits, whatever the other effects are (see Nottale et al. 1997).

One of the easiest ways to check the validity of scale relativity is to verify the presence of small bodies on the orbits $N=1$ or $N=2$. It is important to notice that the absence of objects does not mean that the theory is wrong, but could be explained either by a physical mechanism having ejected the bodies, or by the fact that there is a ring of objects too small to be detected. However, the discovery of one or more objects on these orbits would reinforce the theory.

The first orbit, at $0.05 \mathrm{AU}$ from the Sun, if the eccentricity is small, corresponds to a surface temperature of about $1700 \mathrm{~K}$, assuming the same albedo as Mercury. There is a chance that the heating due to the Sun has already evaporated the body. The theory predicts a size which is too low to be detected on this orbit $(7 \mathrm{~km}$ assuming the same density as Mercury). For these reasons, we preferred to concentrate our efforts on the orbit $N=2$ at $0.18 \mathrm{AU}$, where an object will have a size of about $500 \mathrm{Km}$, and whose surface temperature should be around $900 \mathrm{~K}$. If we look at the perturbation of Mercury's orbit by such an object, calculation shows (see Nottale et al. 1997 for details) that the object could have a size up to $1400 \mathrm{Km}$ before perturbing Mercury's perihelion advance more than the current uncertainty in its measurement.

There have been already two investigations on the orbit $N=2$. First, Leak et al. (1987) observed from the ground an area corresponding to $10 \%$ of the orbit, at $3.5 \mu \mathrm{m}$ wavelength. Recently, Durda et al. (2000), visually examined images from the LASCO coronograph aboard SOHO. The outer coronograph C3 has an angular field of 15.5 degrees, centered on the Sun. This corresponds to about $80 \%$ of the extension of the orbit $N=2$, assuming zero eccentricity. Durda et al. found no object in this field, with a maximum sensibility of $V=8$, corresponding to objects ranging from 20 to $60 \mathrm{~km}$ diameter, depending on the values used to calculate albedo and density. A ring of many small objects is may be more probable than a single object, because the mass is too small for its accretion.

We decided in 1996, to search for vulcanoids on the orbit $N=2$, by analyzing the images produced by LASCO. In the following we discuss our data processing technique, which is quite different from that of Durda et al.

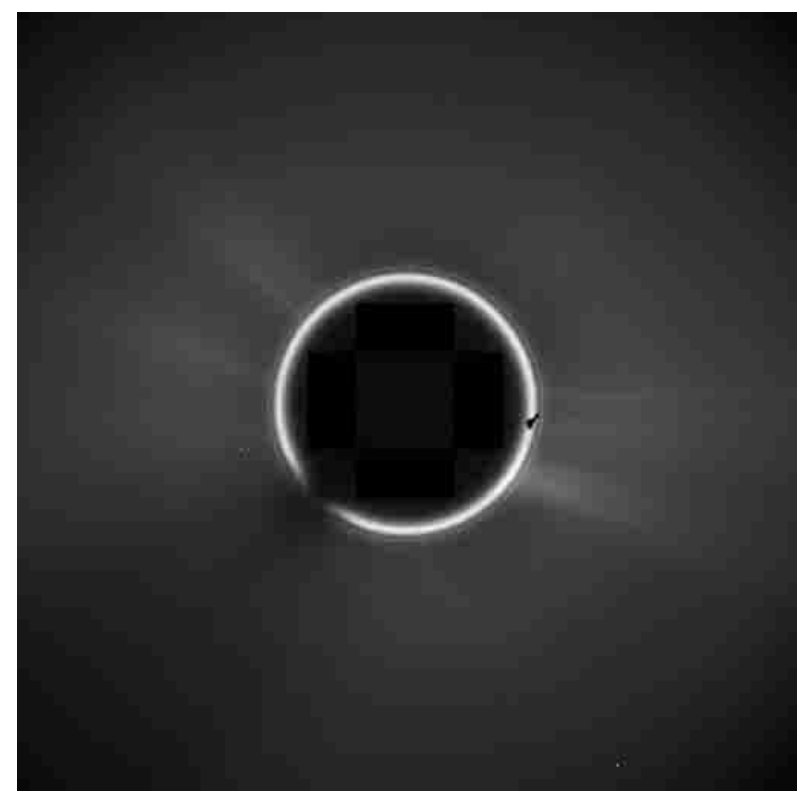

Fig. 2. C2 coronograph raw image. Date: 05/31/1998. Time: 20:28:35 UT. Exposure: 25.1 s. Filter: orange. At the center, the Sun is hidden by the occulter

\section{Data processing technique}

\subsection{LASCO instrument}

LASCO (Large Angular Spectroscopic Coronograph) is a set of three coronographs, and one of the 11 instruments installed on the joint NASA/ESA SOHO spacecraft launched in December 1995 (Brueckner 1995). The spacecraft is located on the Sun-Earth Lagrange point, i.e. on a $600000 \mathrm{~km}$ large stationary orbit at about 1.4 million $\mathrm{km}$ from the Earth, towards the Sun. LASCO produces visible wavelength images of the solar corona from 1.1 to 32 solar radii, oriented along the polar solar axis. The inner coronograph $\mathrm{C} 1$ has also some spectrometric capabilities.

\subsection{LASCO data}

The coronographs $\mathrm{C} 2$ (field of view from 2.0 to $6.0 R_{\odot}$ or 2.91 degrees) and C3 (field of view from 3.7 to $32 R_{\odot}$, or 15.56 degrees), mostly produces CCD images measuring $1024 \times 1024$ pixels, with an angular resolution of 56 arcsec per pixel. Once a day they also produce a set of polarized as well as a set of color filtered images of $512 \times 512$ pixels, having an angular resolution of 112 arcsec per pixel. Figure 2 shows a $\mathrm{C} 2$ image taken with an orange filter, and Fig. 3 shows a C3 image taken with a polarized filter. These polarized and color filtered images have the advantage to being taken in rapid succession, every 4 min or so. We used sets of such successive images to eliminate cosmic rays impacts. Each day we took 4 polarized images in rapid succession and then 12 hours later we took 4 sucessive color-filtered images. Thus, after processing (see below for details) we obtain two images each day free of cosmic rays. We can also retrieve the position and the 


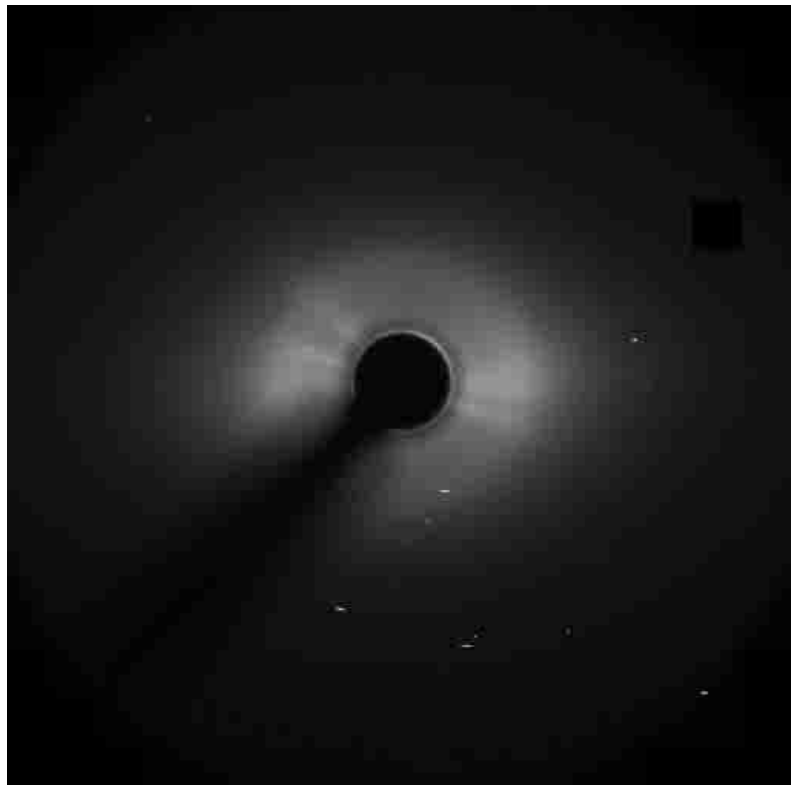

Fig. 3. C3 coronograph raw image. Date: 05/31/1998. Time: 23:00:19 UT. Exposure: 300 s. Filter: orange. Polariser: +60 deg. On the lower-left of the picture one see the finger of the coronograph, an at the center its occulter

velocity of the satellite. We analyzed the data from January to May 1998, with about $15 \%$ image loss due to satellite problems.

\subsection{Pre-processing}

We performed the following operations on all of the $\mathrm{C} 2$ and C3 images corresponding to the same period of time:

First of all we subtracted the flat-field image from every raw image in order to correct the CCD non-uniformity. We also corrected each image for the geometric distortion introduced by the CCD.

Median: Secondly we applied a 2-dimensional median algorithm to each flat-fielded image. This allows us to eliminate the bright background, and so reveal the very faint details of the image. The principle of this operation is the following: on every pixel we attach a square box of 5 by 5 pixels. Next we compute the histogram of the signal's amplitude within the box and take its median value. We then make a second image by giving each pixel the median value which corresponds to it. This way we obtain an image with a backgound that varies slowly over the size of the box. Finally, we subtract this second image from the first one, obtaining an image with only the fast amplitude fluctuations. This means that we have eliminated the corona's bright background and now see the marks left by stars, cosmic ray impacts etc. with equal sensitivity over the whole field. This operation is very efficient and permits, as we will see below, the detection of objects with a visual magnitude $M_{\mathrm{v}}=9.5$. Figure 4 shows a typical median processed image of $\mathrm{C} 3$.

Cosmic ray removal: Since the number of Cosmic Rays striking the CCD is very large, and generally greater than

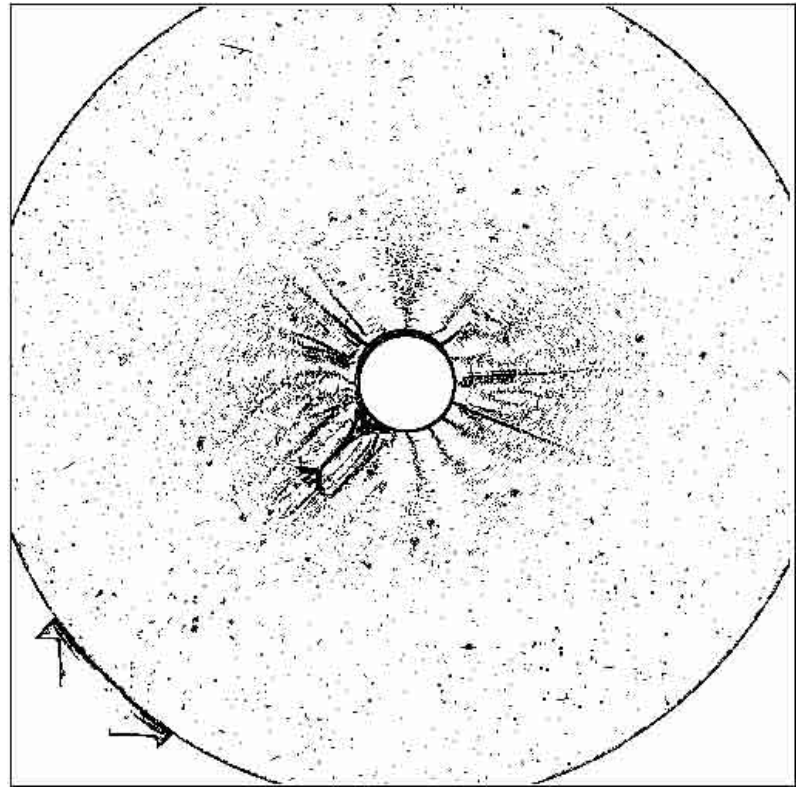

Fig. 4. C3 median processed image. Box size: $5 \times 5$ pixels. The color palette is inverted to improve the visibility. One can see a typical impact of a grazing cosmic ray on the top. Most of the other dots are cosmic rays

100 , it is important to take account of this in order to stand a chance of detecting objects as small as Vulcanoids. We overcome the problem posed by cosmic rays through the use of 4 successive polarized or colored images. The fact that the light is filtered by a polarizer, with different orientations or by a filter of different colors, should not affect very much a Vulcanoid's magnitude. The motion of the Vulcanoids is slow enough that their image stays within the same pixel during the delay corresponding to the 4 successive images. However, a cosmic ray impact appears only once on a unique image. If we compare the 4 successive images, we must see each cosmic ray only in one image, while the slowly moving objects must appear in every image. We have written some software to carry out this comparison and produce an image free of cosmic rays (Fig. 5).

After performing this "cleanup" the resulting images for an entire month's observation (about 60 images) are stored as a three dimensional array. Each image occupies a layer of this "cube". Important information such as the date, time of observation, details of the filter and SOHO's position and velocity, is also stored there. The cube's third dimension is time, which makes this a convenient storage configuration for use in animation.

Fixed pixels removal: At this stage of the processing, we detect so-called fixed bright pixels, corresponding to defects of the CCD, and we eliminate them.

Rotation: The images of LASCO are oriented along the solar polar axis. In order to detect objects orbiting around the Sun (see below) we need to position ourselves in a socalled "osculator" frame of reference (see Fig. 11). This is defined to have its origin $S$ at the current position of the satellite, so that $\boldsymbol{O S}$ is the Sun-> satellite vector, $\boldsymbol{v}$ is 


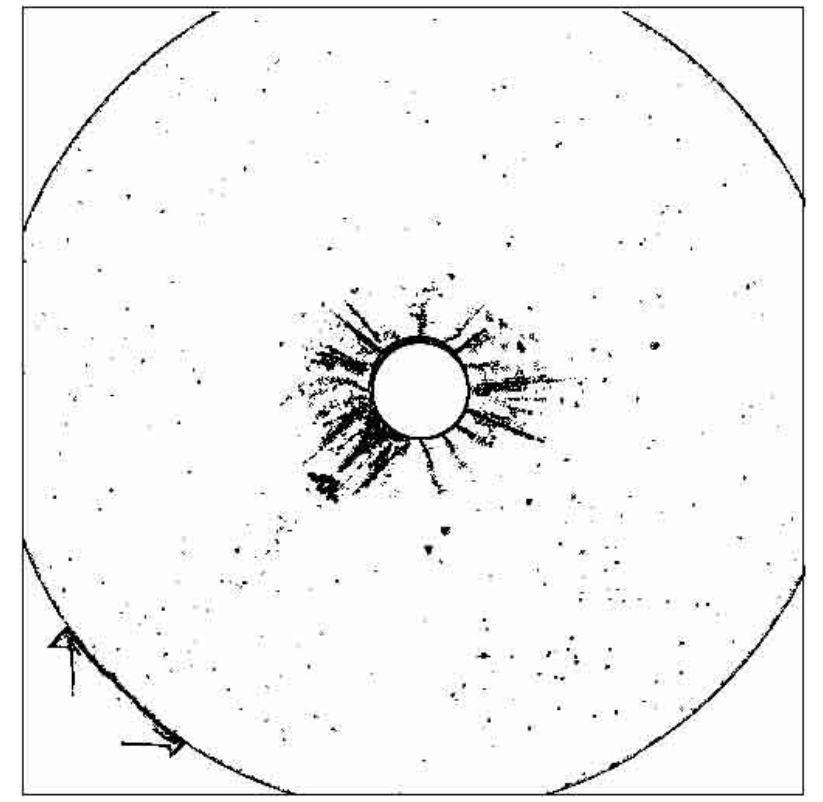

Fig. 5. C3 image resulting from a set of 4 polarized images, after removal of the cosmic rays

the satellite's instantaneous velocity and $\boldsymbol{N}$ is a vector normal to the orbital plane. We rotate each image so that its lower boundary is parallel to $\boldsymbol{v}$.

Stellar removal: In order to remove the stars from the images, we compared them with the SAO catalog for the corresponding field. We have statistically analysed of the result of this operation. It turns out that we detect (and eliminate) $100 \%$ of the stars up to the magnitude $M_{\mathrm{v}}=7$ of the catalog, everywhere on the field. For higher magnitudes the percentage decreases, becoming almost $0 \%$ for magnitude 9.5. This means that the sensitivity of the camera and our processing method allows to detect objects up to magnitude $M_{\mathrm{v}}=7$. Figure 6 shows an image cleaned from the cosmic rays and from the stars.

Composition C2 + C3: To widen the field containing information in the central part of the images, around the occulter, we have applied all the previous operations to the $\mathrm{C} 2$ images. Then, we have added the $\mathrm{C} 2$ to the $\mathrm{C} 3$ images, after adjustment of their scale (Fig. 7).

Large defect removal: At this step of the processing there are still some large black objects having nothing to do with Vulcanoids. There is some trace of the finger of the coronograph, as well as a ring corresponding to the outer limit of the image. But, more embarrassing, around the occulter, there is always a zone corresponding to high activity in the corona, probably matter ejection, and also noise resulting from the image processing. We have written a routine which eliminates the objects having a size larger than $3 \times 3$ pixels (Fig. 8 ).

Refining: The last operation we do is intended to decrease the computer time at the subsequent step of orbit detection (see below). The objects on the images do not have all the same brightness, and often a brighter object corresponds to larger objects in terms of number of pixels. Our routine of automatic orbit detection will

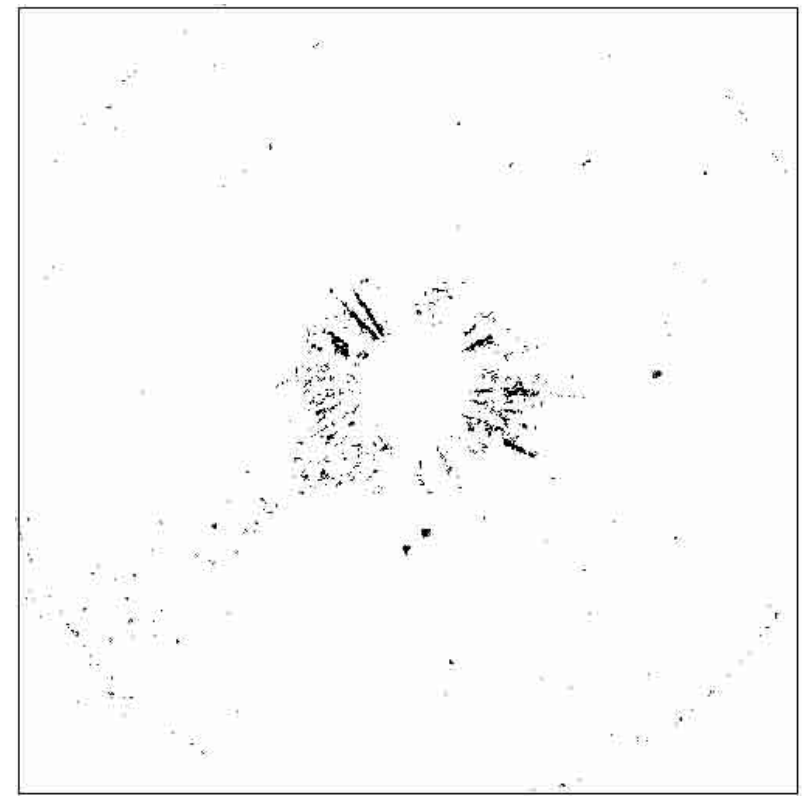

Fig. 6. Image with stars removed

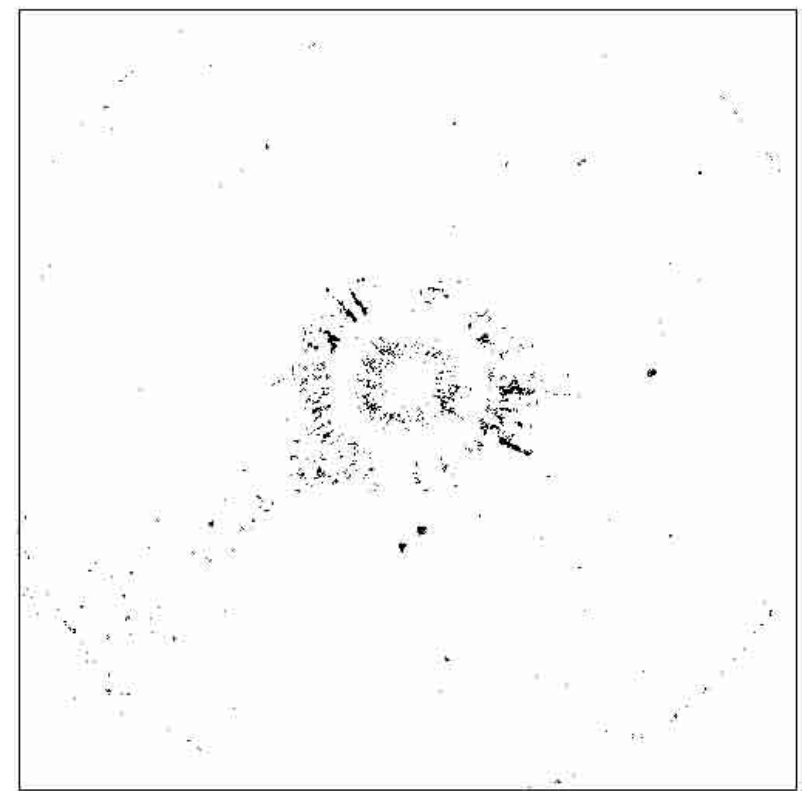

Fig. 7. Composition of $\mathrm{C} 2$ and $\mathrm{C} 3$

analyze each illuminated pixel as an individual object, which greatly increases the number of possible combinations, and consequently the computing time. But, we know that a Vulcanoid can not have a size larger than one pixel on the images. So, to simplify the next operation, we replace every object by one illuminated pixel at its barycenter (Fig. 8).

We finally obtain images which still contain about 30 bright pixels, among which we have to detect possibly real astronomical objects. Most of the information contained in the images is probably due to noise coming from the CCD and from the processing technique. But, we have no way to eliminate it. 


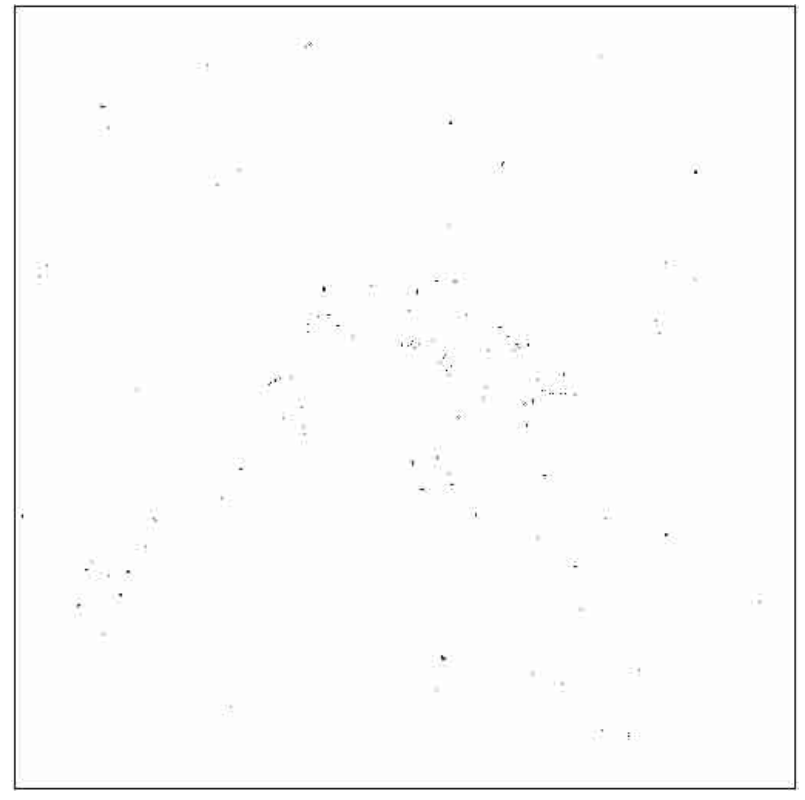

Fig. 8. Image cleaned of large defects

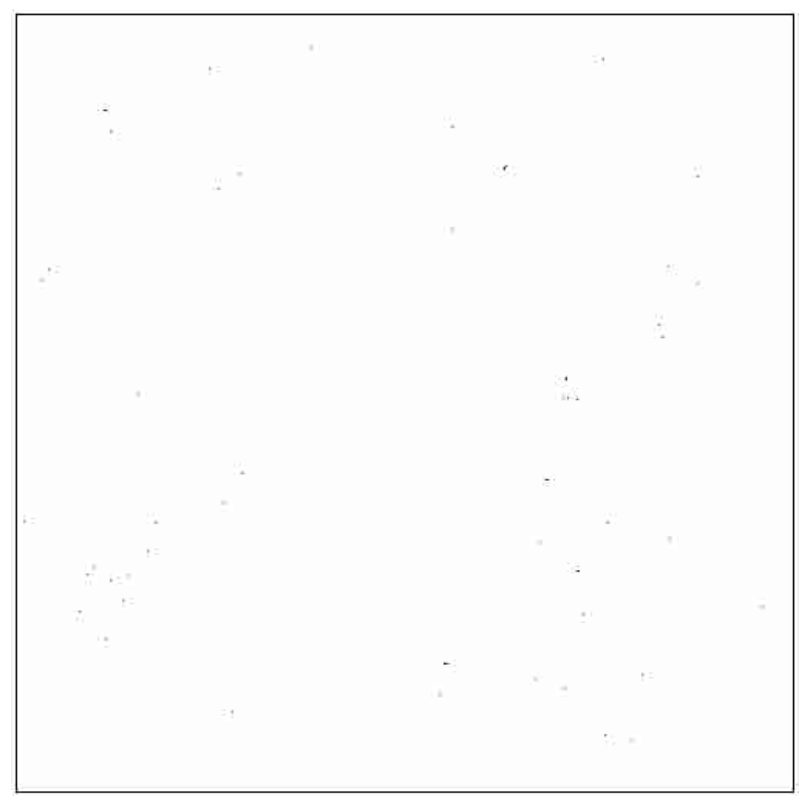

Fig. 9. Refined image

\subsection{Orbit detection}

\subsubsection{Visual detection}

The first thing we do with the final images is to view the entire set for a month using animation. In this way we try to detect possible unknown objects. We see all of the planets up to the magnitude of Uranus. Occasionally we detect Neptune but never Pluto which is too faint. For the period from January to May 1998 we have seen no new object in the field of observation with this method.

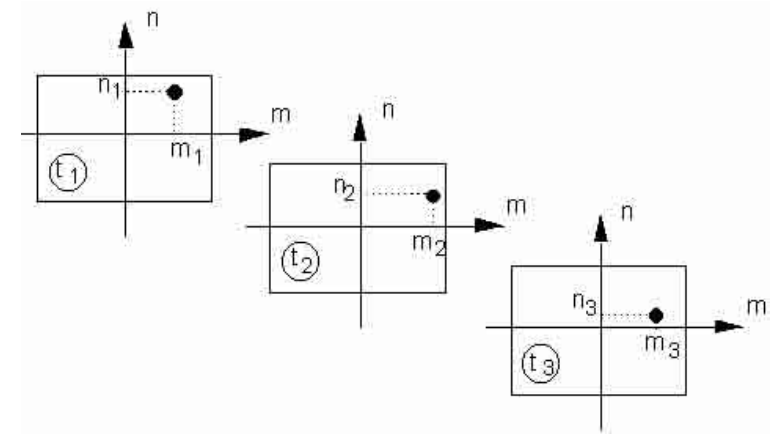

Fig. 10. Position of the image of an asteroid in the LASCO field at tree different times

\subsubsection{Computer automated detection}

The method we use for automatically detecting new objects moving around the Sun consists in analysing successive cleaned-up images to see it there exists a combination of points that could correspond to a true orbit.

Suppose there are three successive observations, which we call a triad, made at times $t_{1}<t_{2}<t_{3}$ (see Fig. 10).

We select the triads corresponding to realistic orbits by limiting the variation of the area scanned by the radius vector, by limiting the speed of the object, and by minimizing the concavity of the apparent orbit. The apparent positions $\left(m_{j}, n_{j}\right)$ with $j=1 \ldots 3$ allow us in theory to access to the six orbital parameters $O=\left\{a, e, i, t_{0}, \Omega, \chi\right\}$ by expressing the radius vector $\rho$ of the object from its components by using Newton's law of motion:

$\frac{\mathrm{d}^{2} \boldsymbol{\rho}}{\mathrm{d} t^{2}}=-G M \frac{\boldsymbol{\rho}}{\rho^{3}}$.

The above symbols are defined as follows:

$G$ is the gravitational constant, and $M$ the solar mass;

$a$ : semi-major axis;

$e$ : eccentricity;

$i$ : inclination of the orbital plane;

$\chi$ : longitude of the perihelie;

$\Omega$ : longitude of the ascending node;

$t_{0}$ : date of node passage.

The simplest reference frame is related to the radius vector of the satellite and its velocity (Fig. 11). We call it the "osculator frame". The Sun is at the center $O$, and the satellite is at $S$ at the position hecx, hecy, hecz. XOY is the ecliptic plane. The trihedron $X Y Z$ is determined by $\boldsymbol{O} \boldsymbol{X}$, oriented towards the vernal point $\gamma$. The vertical plane on the figure is normal to $\boldsymbol{O S}$ and contains $O$. It is called the image plane because it is parallel to the plane of the camera CCD. $S^{\prime}$ is the projection of $S$ to the ecliptic plane, and determines the angle $\Psi$. $\boldsymbol{O} \boldsymbol{\omega}$ is the polar vector of the Sun, and the inclination of the solar equator with the ecliptic is $\theta=7.52 \mathrm{deg}$ and the longitude of the ascending node is $\alpha=74^{\circ} 22^{\prime}+84 T$ ( $T$ is given in centuries, origin 1900.0). In 1997, $\alpha=75.72^{\circ}$.

$\boldsymbol{\omega}^{\prime}$ is the projection of $\boldsymbol{\omega}$ to the image plane. The angle $\beta$ is the projected rotation angle, i.e. the angle with $\boldsymbol{O} \boldsymbol{Z}^{\prime}$ 


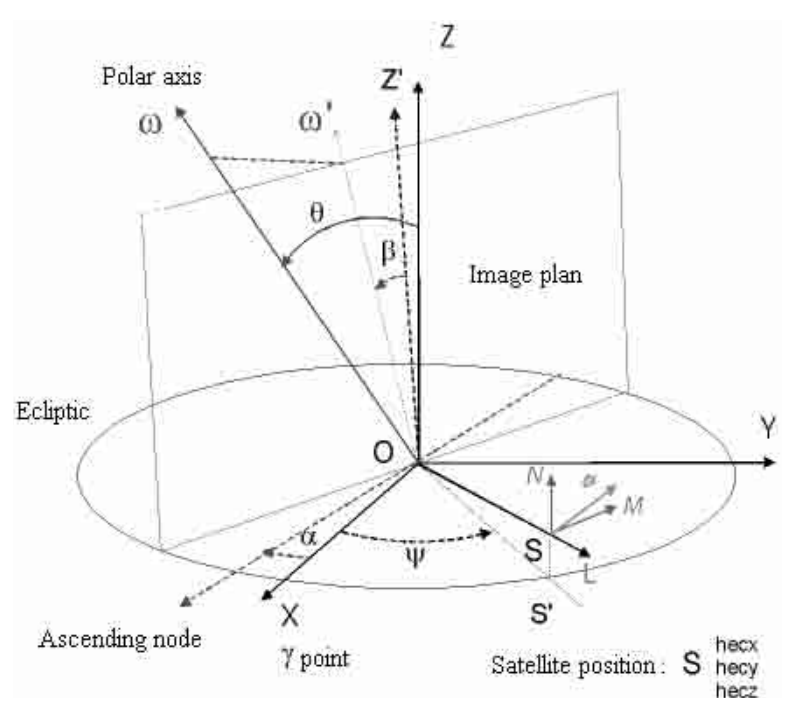

Fig. 11. Coordinate system of the osculator frame

which is the projection of $\boldsymbol{O Z}$ to the image plane. The trihedron $M N L$ is defined as following:

- $\boldsymbol{S L}$ is colinear with $\boldsymbol{O S}$;

$-\boldsymbol{S M}$ is in the osculator plane containing $\boldsymbol{O S}$ and the velocity vector of the satellite $\boldsymbol{v}$;

$-\boldsymbol{S N}$ completes the trihedron.

The methods proposed by Laplace, Gauss, and Olbers, optimized for asteroids observed close to opposition, are not suitable to treat the problem of objects close to the Sun. We have developed another method whose first step consists in fitting the apparent trajectory by a quadratic law in time:

$m(t)=a+b\left(t-t_{2}\right)+c\left(t-t_{2}\right)^{2}$

$n(t)=A+B\left(t-t_{2}\right)+C\left(t-t_{2}\right)^{2}$.

The knowledge of the six constants $(a, b, c, A, B, C)$ obtained by fitting this law to the measured values $M=$ $\left\{m_{1}, n_{1}, m_{2}, n_{2}, m_{3}, n_{3}\right\}$ allows a first estimation of the orbital parameters $O_{1}$. Figure 12 shows the projection of the image to the focal plane of LASCO. However, if one computes backwards the apparent positions at the instants $t_{1}, t_{2}, t_{3}$ from the orbit $O_{1}$, one does not find exactly the starting positions $M$. The difference comes from the inadequacy of the quadratic form for representing. One can verify it by noticing that the apparent acceleration $\{2 c, 2 C\}$ conserves a constant direction in the field, while it should always point to the image of the Sun, if one neglects the acceleration of the reference frame.

A second iteration of the program is then necessary. Let us introduce an operator $Q$ which transforms the sixdimensional position vector $M$ into six-dimensional orbital parameter vector $O$ :

$O=Q(M)$.

The inverse transformation is given by an operator $P$ :

$M=P(O)$

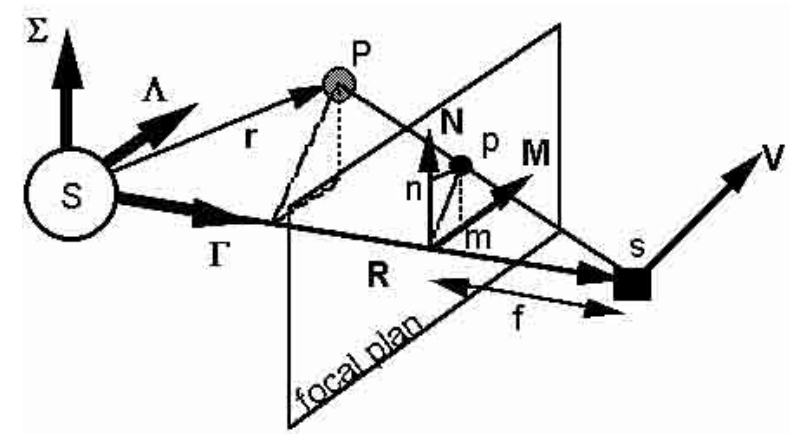

Fig. 12. Projection of the image of an asteroid on to the focal plane of LASCO

$P$ and $Q$ should be reciprocal, but there is a small difference

$\delta M=P(Q(M))-M$.

One applies to the apparent coordinates the correction $\Delta M$ such as

$P(Q(M+\Delta M))-M=0$.

The correction $\Delta M$ is small and a first order development is enough. We give successively to each component of $M$ a variation of one pixel $\Delta M_{i}$, and we observe the corresponding variation $\delta M_{i}$. One then computes the derivation matrix of $\delta M$ versus $M$, whose inverse allows the calculation of the correction $\Delta M$ such that the orbit $O^{\prime}=Q(M+\Delta M)$ verifies the relation $P\left(O^{\prime}\right)=$ $M$. The components of $O^{\prime}$ are solutions of the problem. Simulations show that one iteration is enough to converge to an accuracy of the components of $M$ lower than one pixel, which is the accuracy of the apparent positions measured in the field of LASCO.

It is possible for there to be no solution for the equation $O=Q(M)$. For example, a triad which does not correspond to a Keplerian orbit. There could also be two solutions, expressing the indeterminacy of the position in front or behind the Sun. The double solution corresponds to the maximum elongation.

\section{Results}

We have applied a fully automatic detection process to data obtained between January and May 1998. Figure 13 shows a typical result for February and gives the values of the semi-major axis as a function of the triads. There are about 2500 triads but only 200 or so of them lead to a real solution. As one can see, there is no obvious concentration of points around a given value of the semi-major axis. The same happens with the other orbital parameters. Even in the "cleaned up" images there are still too many points arising from noise produced during the processing of data. There is also a very high density of noise close to the occulter border coming partially from matter ejection around the solar equator. With this automatic method, 


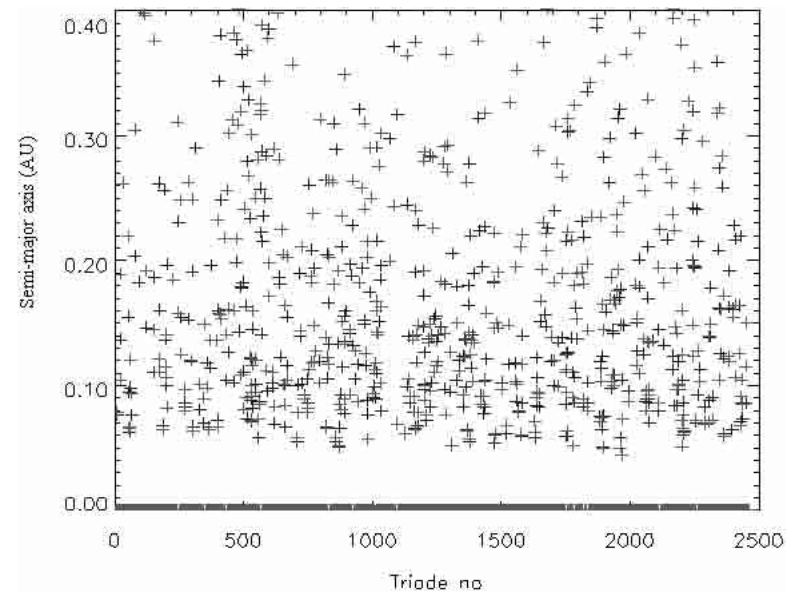

Fig. 13. Semi-major axis versus triad number

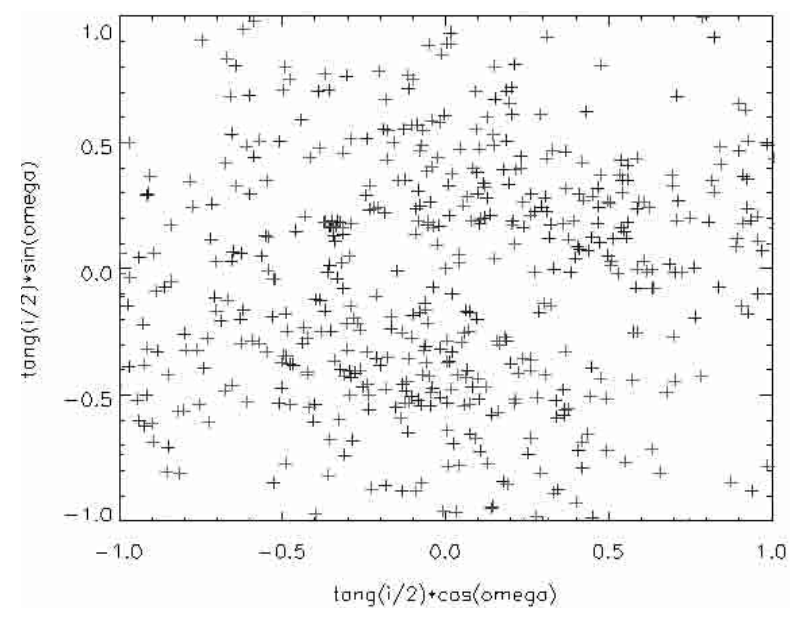

Fig. 14. Longitude of ascending node versus inclination in stereographic projection

we are unable to detect even Mercury (semi-major axis of $0.35 \mathrm{AU}$ ) even though we easily detect it visually on the images. Jupiter and Uranus, which are also in the field of view, have semi-major axes too large to be seen in Fig. 13.

Figure 14 shows a stereographic projection from the pole, for February 1998, of parameters $\tan (i / 2) \cdot \sin (\Omega)$ versus $\tan (i / 2) \cdot \cos (\Omega)$. Such a representation of the data is a cross section of the six dimensional space of the orbital parameters, and it should more easily reveal a concentration of points, expressing an eventual orbit.

In fact, the values in this coordinate system are very close to zero for all the planets of the solar system, explaining why we don't detect Mercury or Jupiter. We see on the diagram that we are not able to detect any other object because there is too much noise.

Finally, automatic detection seems not to be as efficient as visual detection. The detection limit is first given by the sensitivity of the detctor, and we have calculated that it corresponds to the magnitude $m_{\mathrm{v}}=7$. Visual detection is just limited to this value, but automatic detection is limited by the complexity to process the noise close to the Sun.

\section{Conclusion}

During the period of observation from January to May 1998, we detected no new object with magnitude greater than 7 in the fields covered by the C2 and C3 coronagraphs. If Vulcanoids have the same density and albedo as Mercury, then this means that there is no object with a diameter greater than about $60 \mathrm{~km}$ revolving around the Sun within the area we investigated. This is consistent with the results reported by Durda et al. (2000).

However, it should be noted that our method of "cleaning" still leaves the images with a lot of noise, especially in the ecliptic plane. The inclination $i$ of the orbital planes of the known planets is less than 7 degrees. If the object we are searching for on the $N=2$ orbit has a comparable inclination angle, then an important part of its trajectory will probably be drowned in the "ecliptic noise".

Another possible explanation for our negative result is that the mass predicted on orbit $N=2$ by scale relativity theory, namely about 1 hundreth of Mercury's mass, is not that of a single object but is distributed over several smaller objects. If this is so then there is no hope of detecting these objects by studying LASCO images.

The best way of verifying whether there is matter present on the $N=2$ orbit is probably to make measurements in situ. The European Space Agency project "Solar Orbiter" might be an efffective means for doing this.

Even though there is still no direct confirmation of scale relativity theory within the solar system, we ought to mention that 50 per cent of the recently discovered exoplanets are located on $N=1$ and $N=2$ orbits as shown in a recent paper by Nottale et al. (2000).

Acknowledgements. This work was supported by the CNRS. We have benefitted from numerous discussions with Dr. Laurent Nottale (Observatoire de Paris). Dr. Patrick Michel (Observatoire de la Côte d'Azur) kindly provided us with his simulations of orbit stability. Drs. Ph. Lamy and A. Llebaria (Laboratoire d'Astronomie Spatiale) helped with the acquisition of data.

\section{References}

Brueckner, G. E., Howard, R. A., Koomen, M. J., et al. 1995, Solar Phys., 162, 357

Durda, D. D., Stern, S. A., Colwell, W. B., et al. 2000, Icarus, 148 , in press

Evans, N. W., \& Tabachnik, S. 1999, Nature, 399, 41

Leake, M., Chapman, C. R., Weidenshilling, S. J., et al. 1987, Icarus, 71, 359

Lebofsky, L. A. 1975, Icarus, 25, 205

Michel, P., private communication

Nottale, L. 1993, Fractal Space-Time and Microphysics: Towards a Theory of Scale Relativity (World Scientific)

Nottale, L. 1996, Chaos, Solitons and Fractals, 7(6), 877

Nottale, L. 1997, A\&A, 327, 867

Nottale, L., Schumacher, G., \& Gay, J. 1997, A\&A, 322, 1018

Nottale, L., Schumacher, G., \& Lefèvre, E. T. 2000, A\&A, 361, 379

Stern, S. A., \& Durda, D. D. 2000, Icarus, 143, 360

Vokrouhlicky, D. 1999, A\&A, 344, 362 